\title{
Del consumismo al \\ consumo sostenible
}

From consumerism to

sustainable consumption

Recibido: 3 de enero 2013

Evaluado: 2 de marzo de 2013

Aceptado: 10 de abril de 2013

\section{Clemencia Camacho Delgado (Colombia)}

Institución Universitaria Politécnico Grancolombiano

ccamacho27@gmail.com

\section{Resumen}

Este artículo problematiza las características del consumo humano y se apoya de autores como Max Neef, Zigmund Bauman y Barber, para explorar un escenario de consumo sostenible que comprenda la población en relación con el uso de los recursos.

Palabras clave: consumo sostenible, sostenibilidad, mercado, ecosistemas.

\section{Abstract}

In this paper, the author elaborates on the characteristics of human consumption, supported by authors such as Max Neff, Zigmund Bauman and Barber, to explore a sustainable consumption scenario comprising the population in relation to the use of resources.

Keywords: sustainable consumption, sustainability, market, ecosystems. 
Es necesario entender cuáles son las necesidades humanas para abordar el consumo humano. El economista chileno Max-Neef (1993) diferencia las que son propiamente necesidades (existenciales: ser, tener, hacer estar; axiológicas: subsistencia, protección, afecto, entendimiento, participación, creación, ocio, identidad y libertad), de los satisfactores de esas necesidades humanas, y sostiene que no existe una correspondencia biunívoca entre necesidades y satisfactores. Este último puede contribuir al mismo tiempo a la satisfacción de diversas necesidades, y viceversa, una necesidad puede requerir de diversos satisfactores para ser satisfecha. Ni siquiera estas relaciones son fijas. Pueden variar según el momento, el lugar y las circunstancias.

En su libro Desarrollo a escala humana sostiene:

Las necesidades humanas fundamentales son pocas, delimitadas y clasificables, son las mismas en todas las culturas y en todos los períodos históricos. Lo que cambia a través del tiempo y de las culturas es la manera o los medios utilizados para la satisfacción de las necesidades. Cada sistema económico, social y político adopta diferentes estilos para la satisfacción de las mismas necesidades humanas fundamentales. En cada sistema éstas se satisfacen (o no) a través de la generación (o no generación) de diferentes tipos de satisfactores. Uno de los aspectos que define una cultura es su elección de satisfactores. Las necesidades humanas fundamentales de un individuo que pertenece a una sociedad consumista son las mismas del que pertenece a una sociedad ascética. Lo que cambia es la cantidad y calidad de los satisfactores elegidos, y/o las posibilidades de tener acceso a los satisfactores requeridos. Lo que está culturalmente determinado no son las necesidades humanas fundamentales, sino los satisfactores de esas necesidades. El cambio cultural es consecuencia, entre otras cosas, de abandonar satisfactores tradicionales para reemplazarlos por otros nuevos y diferentes (Max-Neef, 1993, p. 144).

Por otra parte, la definición de consumo, según el Diccionario de la lengua española (Real Academia Española, 1970), dice: "gasto de aquellas cosas que con el uso se extinguen o destruye". El economista estadounidense Jeremy Rifkin (2008) al respecto afirma:

En su forma original consumir significaba destruir, saquear, someter, acabar o terminar. Es una palabra forjada a partir de un concepto de violencia $y$, hasta el presente siglo tenía tan solo connotaciones negativas, la metamorfosis del concepto de consumo desde el vicio hasta la virtud es uno de los fenómenos más importantes observados durante el transcurso del siglo XX (p. 201).

El sociólogo, filósofo y ensayista polaco Zigmund Bauman (2000) considera que estamos en una sociedad de consumidores, en la que ser consumidor significa: usar las cosas, comerlas, vestirse con ellas, utilizarlas para jugar y, en general, satisfacer a través de ellas las necesidades y deseos, a más que el dinero media entre el deseo y su satisfacción; ser consumidor también significa apropiarse de las cosas destinadas al consumo: comprarlas, pagar por ellas $y$, de este modo, convertirlas en algo de exclusiva propiedad, con lo 
cual se impide que los otros las usen sin consentimiento.

No obstante, el consumo como tal es el que mantiene nuestra vida, nos la hace placentera y abastece las necesidades de los humanos; todos necesitamos consumir para vivir, esto no es cuestionable. Sin embargo, nos hemos desviado al consumismo que tiene que ver con el exceso, con tomar más, mucho más de lo que necesitamos y, por lo tanto, exigir más recursos de los que el planeta Tierra puede aportar. La presión de comprar más cosas, más nuevas, más sofisticadas está relacionada con la presión de expresar el estatus y la identidad, lo que implica encontrar seguridad al sobresalir y al encajar en el grupo social. El consumo sirve a estos dos, a la cultura del capitalismo y a la mercantilización, hace hincapié en el sobresalir y el encajar por medio de las posesiones y su exhibición. La casa, el carro y el guardarropa, el uso de celulares y tecnología se hacen cada vez más visibles (Speth, 2008).

La palabra consumismo no aparece en el Diccionario de la lengua española de 1970. Sin embargo, está definida en el mismo diccionario de la edición de 2005 con el significado de "afán por comprar bienes indiscriminadamente, aunque no sean necesarios".

En palabras de Barber (2008), "el consumismo privilegia el impulso sobre la deliberación, la gratificación instantánea sobre la gratificación a largo plazo, el narcisismo sobre la sociabilidad, el derecho sobre la responsabilidad, y el ahora sobre el pasado y el futuro" (p. 76).

Dentro de nuestra sociedad, y para lo que estamos aleccionados, no comprar nos hace culpables, significa fallarle al sistema, apagar la economía. ¿De dónde viene esto? Leonard (2010) del capitalismo como modelo económico dominante, con una incesante necesidad de producir ganancias, manteniendo el ritmo frenético de una economía en perpetua expansión y sostenido en la cultura del consumismo. Varios factores inciden según Orr (1999), como la idea de que los recursos de la Tierra son inagotables, el surgimiento del capitalismo moderno, la inteligencia tecnológica y la extraordinaria prodigalidad de Estados Unidos, cuna del consumismo con la seducción publicitaria, las trampas del crédito fácil, la ignorancia sobre los peligros de lo que consumimos, la descomposición de la comunidad, la despreocupación por el futuro, la corrupción política y la atrofia de los medios alternativos que podríamos usar para aprovisionarnos.

El consultor en relaciones industriales Edward Cowdrick (1927) fue de los primeros en referirse al tema, en lo que llamo el nuevo evangelio del consumo, y establecer a este como el camino de salvación para vaciar los almacenes de las empresas, atestados a la sazón de mercancías no vendidas a causa de la superproducción propiciada por los avances tecnológicos en máquinas herramienta que permitían considerables ahorros de mano de obra, y con los niveles de producción en franco crecimiento, lo que consistió en la revolución económica según la cual el estamento empresarial empezó a buscar de forma desesperada nuevas maneras para reorientar la psicología de aquellos que todavía disponían de capital: ya no produciría solo el objeto por vender, sino que inventaría y produciría también el consumidor. El sector publicitario, según Cowdrick (1927), ya sea ajeno a la empresa industrial, ya sea constituido como empresa independiente, viene a ser, literalmente, una industria productora de 
consumidores; de esta manera, la publicidad, en cuanto industria especializada en la producción del consumidor, se apoderó de la gestión activa del consumo, tras incorporarlo a la economía productiva. Desde entonces el consumidor ha venido pasando a convertirse, aceleradamente, en un producto más, y hoy, con el auge de la televisión, ha llegado a serlo en un grado inimaginable. Para crear, dirigir y controlar a los consumidores e incentivar el consumo se desarrollaron técnicas cada vez más audaces de mercadeo o marketing. "El nuevo interés en el marketing reflejaba una creciente consciencia, por parte de la comunidad empresarial, sobre la importancia fundamental del consumidor para el mantenimiento de la economía" (Cowdrick, 1927, p. 88).

El historiador Frederick Lewis Alien resumió esta nueva forma de pensar de la siguiente forma:

El mundo de los negocios, por fin, ha aprendido la importancia del consumidor final. A menos que se le convenza de comprar y comprar pródigamente toda la caravana de automóviles de seis cilindros, los aparatos superheterodinos, los cigarrillos, las barras de labios y los congeladores eléctricos, su mercado quedará bloqueado (Alien, 1964, p. 114).

Después de la Segunda Guerra Mundial, el consumo se convirtió en el eje necesario para soportar la finalidad primordial de la economía de producir bienes de consumo de acuerdo con los lineamientos de Estados Unidos (Seager, 1993). Para los setenta el consumo, económica y culturalmente, se convirtió en forzoso a más de sensato. Como consecuencia, las deudas de los consumidores se han venido incrementado hasta duplicar o triplicar sus ingresos en un sobreendeudamiento, soportadas por los créditos que dan una gama cada vez más rica de posibilidades (Gutiérrez, Capera y Estrada, 2011). Hoy la mayoría de los países en desarrollo tienen una creciente clase de consumidores seguidores de los patrones del consumismo.

Asimismo, la sociedad y sus costumbres cambiaron al ritmo del aumento del consumo y nos enseñamos a pagar la mercantilización de todo tipo de servicios logísticos o de respaldo emocional, entre otros. Esto nos ha convertido en individuos ansiosos e infelices.

New Economics Foundation (2012) publicó la tercera edición de su Índice del planeta feliz, en la que se clasifica el nivel de felicidad de los países según tres factores: el bienestar que los habitantes de una nación dicen tener, la esperanza de vida y la huella ecológica de una nación; esto da la eficacia con que un país convierte sus recursos en bienestar. En este documento, se expresa que es posible vivir una vida larga y feliz con una huella ecológica mucho más pequeña que la hallada entre las naciones de consumo más elevado. Costa Rica ocupó el primer lugar por segunda vez consecutiva en el listado global; este país abolió sus Fuerzas Armadas en 1949 direccionando los fondos a la educación, salud, cultura y otras inversiones que contribuyen a una mejor calidad de vida. En tanto Estados Unidos aparece en el último lugar entre los países industrializados y muestra una diferencia con Costa Rica de una huella ecológica un cuarto mayor, con una vida más corta y menores índices de satisfacción.

El proceso económico del consumo es considerado como una actividad de tipo circular, dado que se produce para poder 
consumir y, a su vez, el consumo genera producción. Es un proceso presente en todas las culturas, pero las características acentuadas que ha tomado en los últimos treinta años han llevado al fenómeno del consumismo.

\section{Bauman al respecto afirma:}

En su etapa presente de modernidad tardía - esta segunda modernidad o posmodernidad—, la sociedad humana impone a sus miembros (otra vez, principalmente) la obligación de ser consumidores. La forma en que esta sociedad moderna moldea a sus integrantes está regida, ante todo y en primer lugar, por la necesidad de desempeñar ese papel; la norma que les impone, la de tener capacidad y voluntad de consumir (Bauman, 2000, p. 233).

En las diferentes sociedades, el consumo estaba íntimamente ligado a la satisfacción de necesidades, lo que limitaba el mercado. La relación tradicional entre la necesidad y su satisfacción lleva al consumo. En el consumismo, esta relación se transforma, por lo que queda, entonces, revestida: la promesa y la esperanza de satisfacción preceden a la necesidad y son siempre mayores que la necesidad preexistente, aunque no tanto que impidan desear los productos ofrecidos por aquella promesa. En realidad, esta última resultará mucho más atractiva cuanto menos conocida resulte la necesidad en cuestión: vivir una experiencia que estaba disponible, y de la cual hasta se ignoraba su existencia, es siempre más seductor, según Bauman (2000).

El consumismo implica que las cosas dejen de existir, por agotamiento total pero más porque pierden su encanto hasta que dejan de despertar nuestros deseos y pierden la capacidad de satisfacer nuestros apetitos.

Se piensa que el crecimiento económic', la medida moderna de que las cosas están en orden y siguen su curso, el mayor índice de que una sociedad funciona como es debido, depende, en una sociedad de consumidores, no tanto de la fuerza productiva del país (una fuerza de trabajo saludable y abundante, con cofres repletos y emprendimientos audaces por parte de los poseedores y administradores del capital) como del fervor y el vigor de sus consumidores. El papel - en otro tiempo a cargo del trabajo- de vincular las motivaciones individuales, la integración social y la reproducción de todo el sistema productivo corresponde, en la actualidad, a la iniciativa del consumidor (Bauman, 2000).

Nos encontramos, entonces, en una sociedad en la cual la economía depende del consumo, y este se logra por la interiorización del espíritu de consumidor que tiene arraigado los ciudadanos.

\section{La población y el uso de recursos}

Hace mil años la población del planeta apenas llegaba a los 310 millones. En América y Oceanía era tan escasa que se puede estimar como nula. Entre África y Europa vivían unos cien millones de personas. Y, al igual que sucede en la actualidad, el groso de la población se situaba en China, India y el resto de Asia. Entonces ¡cómo ha crecido tan rápido la población humana?

Durante siglos el crecimiento fue muy lento. Los nacimientos eran compensados con la alta mortandad y la mortalidad 
infantil era muy alta. A mediados del siglo XIX, uno de cada cuatro niños no alcanzaba el año de edad, mientras que la mortalidad infantil en la actualidad está en torno a las 45 muertes por cada mil nacimientos en el mundo.

La evolución de la población y el crecimiento poblacional son consecuencia de varios factores interrelacionados. La alimentación, la generalización de la higiene, la sanidad, la difusión de medicamentos y, en general, el desarrollo de la tecnología han sido decisivos para el fuerte crecimiento de la población mundial. Los grandes avances de la medicina, unidos a la revolución científica de la química moderna, consiguieron que enfermedades que antes eran letales fueran curables. Ejemplo de ello es el uso de la penicilina, la erradicación de la viruela, entre muchos otros. Todo esto ha ayudado no solo a disminuir la mortalidad infantil, sino también a aumentar la esperanza de vida del ser humano. Desde mediados del siglo XIX hasta 2004, la esperanza de vida de un recién nacido se multiplicó por dos, pasando de 38.3 años a 75.7 años, lo que también facilita la cantidad de personas viviendo de forma simultánea en el planeta Tierra.

Los saltos en la población establecen que de los casi 1000 millones en 1800 explosión demográfica propiciada por la revolución industrial y el periodo en que la actividad humana modificó en gran parte el entorno natural, saltamos a más de 6000 millones en 2000, pese a las dos guerras mundiales, las enfermedades epidémicas en curso, los desplazamientos masivos de población y otros escollos, para llegar a los 7000 millones a finales de 2011(ver figura 1). A partir de 1950, la población mundial ha tenido un alto crecimiento, incluso con una baja natalidad en numerosos países, ya que a la fuerte y constante reducción de la mortalidad se ha unido el aumento generalizado de la esperanza de vida. La denominada revolución reproductiva constata que la reducción del esfuerzo reproductivo supone una alta eficiencia reproductiva, baja natalidad y alta supervivencia de los individuos. En consecuencia, la población mundial ha crecido lentamente a lo largo de la historia, pero este crecimiento se ha acelerado en los últimos doscientos años (Milhaud, 2011).

El uso de la energía almacenada en combustibles fósiles y las energías hidrológicas, el desarrollo tecnológico y los procesos para sintetizar fertilizantes químicos "no solo dio lugar a una inmensa industria global, sino que también inauguró la posibilidad biológica de emprender una expansión masiva del suministro de alimentos en todo el mundo". 
Figura 1: Población mundial entre el año 1 y 2001. Tomado de Sachs, citado por Maddison (2001)

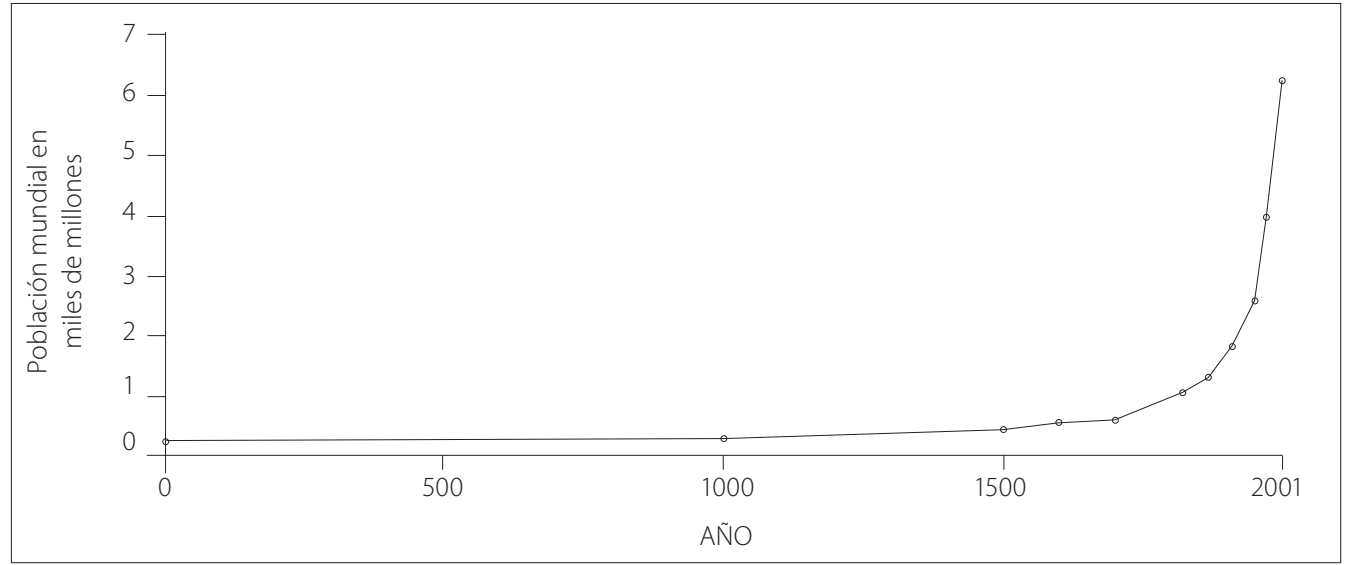

Fuente: Datos tomados de Maddison (2001), Citado por: J. Sachs, Economía para un planeta abarrotado.

El economista estadounidense Jeffrey Sachs opina al respecto:

Este proceso de crecimiento poblacional y económico han llevado a límites inimaginables los niveles de explotación de los recursos naturales. "Haber multiplicado por diez la población humana desde 1750 y haber soportado un crecimiento similar de la producción por persona en el planeta significa que el nivel de actividad económica de la sociedad humana tal vez sea un centenar de veces superior al que era al comienzo de la era industrial. Este incremento se corresponde con el predominio de actividades encaminadas a imponerse a los procesos físicos de la Tierra para satisfacer el consumo humano: la tala de bosques y la producción agrícola, la utilización de la energía, las capturas piscícolas, el empleo de fertilizantes químicos, las presas y los desvíos de cursos fluviales, la construcción de carreteras y muchas otras cosas. No es de extrañar, pues, que los sistemas terrestres de los que depende nuestra existencia se hayan alterado de muchas formas adversas e inesperadas (Sachas, 2008, p. 204).

Los incrementos en la población, a partir de la revolución industrial, los cambios en la producción, el consumo y la economía, se pueden percibir al analizar los registros históricos de productos particulares. Estos se presentan en la figura 2 con valores históricos, entre 1700 y 2000 de la población, el producto interno bruto (PIB), el número de vehículos automotores y el consumo de energía.

Puede observarse cómo a partir de la revolución industrial se presentaron incrementos de la población y el desarrollo económico (PIB) en forma paulatina. A partir de la finalización de la Segunda Guerra Mundial el crecimiento se aceleró en forma notable, como puede verse en la figura 2. El consumo de energía y la fabricación y consumo de productos industriales, representado en la figura 2 Los automotores, sigue muy bien esta tendencia (Steeffen, 2005). 
Figura 2: Rata de cambio en algunos aspectos de la actividad humana. Tomado de Steeffen et al. (2005).
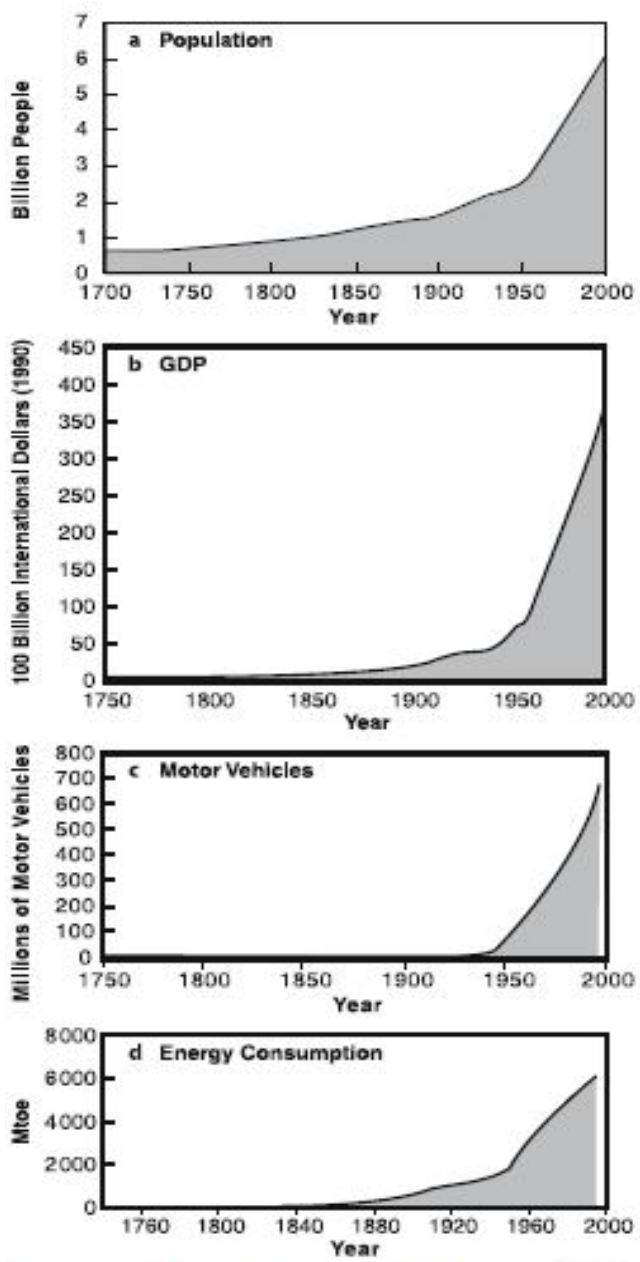

Fig. 1.6. Rate of increase in many spheres of human activity for the last 300 years: a population (US Bureau of the Census 2000): b world economy (Nordhaus 1997); C motor vehicles (UNEP 2000): and denergy consumption (Klein Goldewijk and Battjes 19.97)

El crecimiento poblacional, que ya llega a más de 7000 millones ${ }^{1}$, aunado al ingreso de un gran número de habitantes

\footnotetext{
Las estimaciones de la ONU (basadas en estadísticas) calculaban que, aproximadamente, el 30 de octubre de 2011 el planeta alcanzaría la cifra de 7000 millones de habitantes.
}

de países de economías emergentes, como China, la India, Brasil, entre otras, al consumo mundial crean una demanda cada vez mayor de productos, la cual conlleva la explotación de los recursos y sus problemas asociados a un crecimiento carente de control.

\section{Algunas cifras}

La actividad humana, sobre todo la del último siglo, ha venido cambiando los valores globales del clima, los contenidos de los gases atmosféricos, el porcentaje de los bosques, entre otros factores. La pregunta que debemos contestar es si estos cambios pueden ser soportados por los sistemas de la Tierra o nos llevaraá a una sin salida.

Las evidencias de la crisis ambiental son ahora tan abundantes que solo quienes están seriamente comprometidos con la negación continúan refutando los datos fácticos. Mientras los economistas y los políticos tradicionales parecen ciegos a los patentes límites físicos, hace ya varias décadas que ecologistas, científicos, académicos y demás vienen expresando su preocupación.

Hay cientos de libros e informes, provenientes de incontables fuentes confiables y fidedignas, que documentan el estado actual del planeta. Annie Leonard (2010) presenta los siguientes datos a destacar al respecto:

1) En julio de 2009 ya había 387.81 partes por millón (ppm) de dióxido de carbono $\left(\mathrm{CO}^{2}\right)$ en la atmósfera. Científicos destacados de todo el mundo han establecido la cantidad de $350 \mathrm{ppm}$ como el nivel máximo que puede contener la atmósfera para que el planeta permanezca tal como lo conocemos. 
2) En todos los cuerpos que se analizan hoy en día en cualquier lugar del mundo, incluidos los bebes recién nacidos, aparecen sustancias químicas industriales y agrícolas de carácter tóxico.

3) La contaminación del aire en interiores mata a 1.6 millones de personas por año; el aire exterior se lleva otras 800000 vidas anuales.

4) Aproximadamente, una quinta parte de la población mundial — más de 1200 millones de personas - padecen escases de agua, recurso que se torna cada vez más escaso.

5) La desigualdad global de ingreso es pasmoso. En la actualidad, $1 \%$ más rico del mundo concentra tantas riquezas y cosas como $57 \%$ más pobre.

La contradicción entre el sistema económico, que busca que cada vez se produzca y consuma más, y el sistema global que rige los procesos de nuestro planeta es evidente. Tenemos un subsistema como la economía que crece de forma continua en el interior de un ecosistema de tamaño fijo, el planeta Tierra. Esto lleva a que el expansivo sistema económico en que nos movemos esté alcanzando los límites de la capacidad que tiene el mundo para sostener la vida. Esto se agrava con el continuo crecimiento de los países, sobre todo, de aquellos, como China y la India que están alrededor de $10 \%$ anual. Annie Leonard dice al respecto:

Con la generación actual de volúmenes mundiales de bienes y servicios ya estamos produciendo más de cinco veces (cerca de seis, en realidad) el nivel de emisiones de $\mathrm{CO}^{2}$ que necesitaríamos reducir hacia 2050 para evitar el caos climático total (2010).
Figura 3: Respuesta del sistema terrestre a la presión de la actividad humana. Tomado de Steeffen et al. (2005).
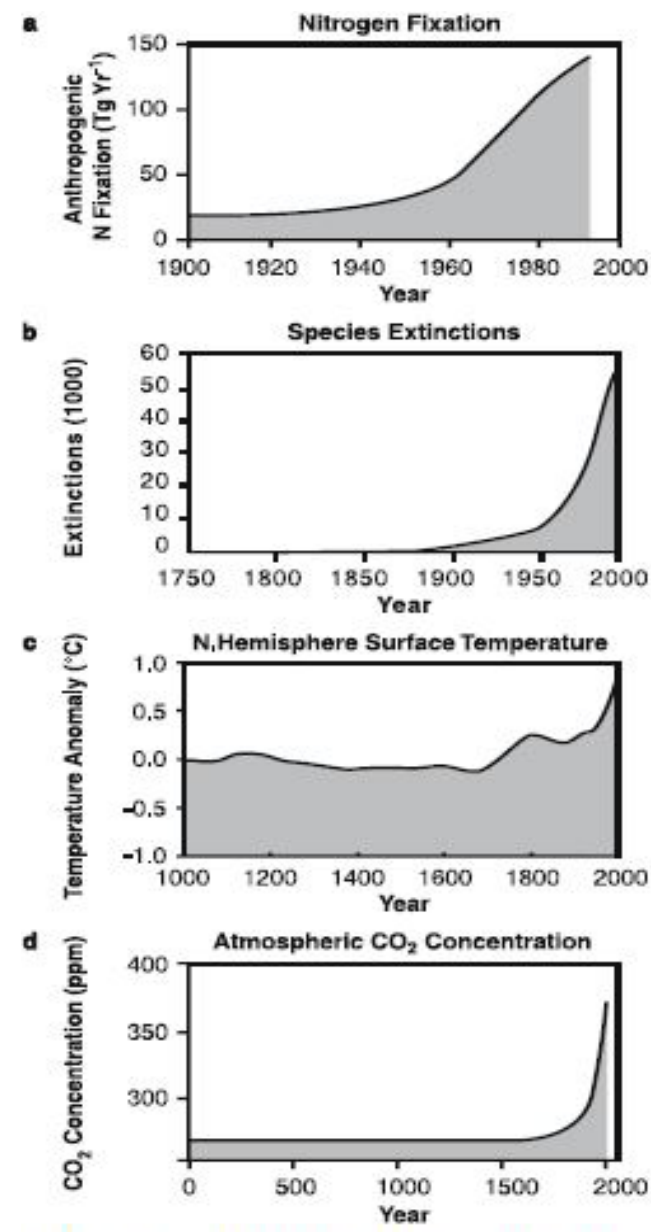

Fig. 1.7. Responses of the Earth System to increasing pressure from human activities: a nitrogen fixation (Vitousek 1994 ); b species extinctions (Smith 2002); $\mathrm{C}$ northern hemisphere surface temperature (Mann et al. 1999): and d atmospheric $\mathrm{CO}_{2}$ concentration (adapted from Keeling and whorf 2000)

En la figura 3, puede verse cómo la concentración de $\mathrm{CO}^{2}$, la fijación del nitrógeno $^{2}$ y la temperatura ambiental

2 Cerca de $60 \%$ del nitrógeno contenido en los fertilizantes no llega a ser absorbido por las plantas y es arrastrado a ríos, lagos, acuíferos y aguas costeras a las que contamina por eutrofización (el exceso de nutrientes agota el oxígeno de las masas de agua) y 
media se han incrementado en forma evidente, debido a la actividad humana en la explotación, producción, consumo y disposición de bienes y recursos naturales. Asimismo, el número de especies extinguidas por cambios en el hábitat, contaminación o sobreexplotación se ha incrementado en forma notoria en el último siglo y tiene un ritmo que, es por decir lo menos, preocupante.

Estamos consumiendo la biodiversidad y los ecosistemas del mundo a un ritmo insostenible, y esto ya está empezando a tener graves consecuencias socioeconómicas. Si queremos encontrar soluciones a los problemas que nos afectan, debemos entender, primero, qué es lo que le está pasando a la biodiversidad y a los ecosistemas, y cómo estos cambios afectan a los bienes y servicios ${ }^{3}$ que nos suministran.

Algunas de las evidencias más claras de la presión que estamos ejerciendo sobre la naturaleza provienen de servicios, tales como los peces silvestres y el agua dulce. Siempre hemos confiado en que los sistemas de la Tierra reemplazarían las cantidades que tomábamos. Hemos considerado a esos servicios como regalos, tras creer que la única limitación para usarlos era la tecnología disponible y el esfuerzo requerido para hacer uso de ellos (Comisario de Medio Ambiente, Comisión Europea, 2008).

puede provocar la muerte de flora y fauna. El óxido de nitrógeno, gas de efecto invernadero, es unas 300 veces superior al dióxido de carbono en sus efectos sobre el calentamiento del planeta.

3 Los servicios ecosistémicos son los servicios que la naturaleza provee a las personas. Entre otros incluye: alimentos, agua dulce, madera, regulación del clima, protección frente a peligros naturales control de la erosión, ingredientes farmacéuticos y actividades recreativas.
En el caso de ambos servicios, las luces rojas de alarma están encendidas, con fuertes indicios de que en muchos lugares hemos sobrepasado el punto en que la naturaleza puede reabastecer los suministros.

Como ejemplo a las consideraciones anteriores y sobre los ecosistemas, a continuación se presenta un resumen de varios autores y de la significativa Declaración del Consejo de la Evaluación de los Ecosistemas del Milenio en el documento sobre capital natural y bienestar humano: estamos gastando más de lo que poseemos, en la que ponen de manifiesto en cuanto a los recursos de la Tierra y la prestación de sus servicios lo siguiente:

\section{Extracción de agua y represado:}

1) La cantidad de agua extraída de los ríos y lagos para el riego, el uso doméstico y la industria se duplicó en los últimos cuarenta años.

2) Los seres humanos ahora utilizan entre 40 y $50 \%$ del agua dulce que llega a los cauces y lagos a los que tienen acceso la mayoría de la población.

3) En algunas regiones, como en Oriente Medio y el Norte de África, los seres humanos utilizan $120 \%$ del suministro renovable (mediante el recurso al agua subterránea que no se recarga).

4) Entre 1960 y 2000, la capacidad de almacenamiento de agua en las presas se cuadruplicó y, como resultado de ello, se calculó que el agua almacenada era entre tres y seis veces más que la que corría por los cauces de los ríos (sin incluir el agua de los lagos naturales). 


\section{Conversión y degradación:}

1) En los treinta años que transcurrieron después de 1950, se destinaron más nuevas tierras al cultivo que en los ciento cincuenta años transcurridos entre 1700 y 1850 , y en la actualidad cerca de un cuarto (24\%) de la superficie terrestre ha sido transformada en sistemas cultivados.

2) Desde cerca de 1980, se ha perdido aproximadamente $35 \%$ de los manglares y se destruyó $20 \%$ de los arrecifes de coral del mundo, y otro $20 \%$ ha sido seriamente degradado o destruido.

\section{Pesquerías:}

1) Por lo menos una cuarta parte de las poblaciones de peces marinos está siendo sobreexplotada.

2) La cantidad de pescado extraído por los seres humanos aumentó hasta la década de los ochenta, pero ahora está bajando debido a la merma de las poblaciones de peces.

3) En muchos lugares del mar, el peso total de los peces disponibles para ser capturados es menos de la décima parte del que se disponía antes de que comenzara la pesca industrial.

4) Las pesquerías en agua continentales, de especial importancia porque brindan una dieta de calidad para los pobres, también están mermando debido a la sobrepesca, los cambios en el hábitat y la extracción de agua dulce.

5) Las actividades humanas generan ahora más nitrógeno biológicamente disponible que el que producen todos los procesos naturales juntos, y más de la mitad de todo el fertilizante a base de nitrógeno fabricado hasta ahora (la producción comenzó en 1913) ha sido aplicada desde 1985.
6) El flujo de nitrógeno hacia los océanos se ha duplicado desde 1860 .

7) El uso de fertilizantes a base de fósforo y la tasa de acumulación de fósforo en los suelos agrícolas aumentó cerca de tres veces entre 1960 y 1990. Si bien el ritmo de ese uso ha declinado un poco desde entonces, el fósforo puede permanecer en los suelos durante décadas antes de entrar en el medio ambiente más amplio.

8) En los últimos trescientos años, la masa forestal mundial se ha reducido aproximadamente $40 \%$. Los bosques han desaparecido totalmente en 25 países y otros 29 han perdido más de $90 \%$ de su cubierta forestal. Además, el declive continúa (Comisario de Medio Ambiente, Comisión Europea, 2008).

9) Desde 1900, se han destruido en torno a $50 \%$ de los humedales del mundo. Mientras que este fenómeno tuvo lugar en los primeros cincuenta años del siglo XX en los países del Norte, desde los ańos cincuenta la destrucción se ha centrado en los humedales tropicales y subtropicales, que están sometidos a una presión cada vez mayor para destinarlos a otros usos (Moser, Prentice y Frazier, 1996).

10) En torno a $30 \%$ de los arrecifes de coral — que han sido una fuente de biodiversidad mayor incluso que los bosques tropicales - se han visto gravemente dañados, debido a la pesca, la contaminación, las enfermedades y la decoloración del coral (Wilkinson, 2004).

11) En las últimas dos décadas, ha desparecido $35 \%$ de los manglares. Algunos países han perdido hasta $80 \%$ de los manglares debido a su conversión para explotaciones de acuicultura, a 
la sobreexplotación y a las tormentas (Evaluación de los ecosistemas del milenio, 2005).

12) Se calcula que, debido a la actividad humana (factores antropogénicos), el ritmo de la extinción de especies es mil veces superior al ritmo "natural" propio de la historia de la Tierra (Evaluación de los ecosistemas del milenio, 2005).

\section{La sostenibilidad del sistema}

El reto de la sociedad actual está en satisfacer la demanda mundial de alimentos, energía, agua, medicamentos y materias primas, y minimizar, al mismo tiempo, los efectos adversos sobre la biodiversidad y los servicios ecosistémicos.

Pero ¿cómo llegar a ser sostenibles? El Concejo Empresarial Colombiano para el Desarrollo Sostenible, establece cuatro puntos principales

1) control del crecimiento de la población;

2) redistribución de la riqueza y acceso a la tecnología;

3) cambio de los patrones de consumo;

4) ecoeficiencia.

En relación con el tercer punto, la Organización de las Naciones Unidas para la Agricultura y la Alimentación dice al respecto:

El desarrollo sostenible requiere cambios en los patrones de consumo y producción, mecanismos de gobernabilidad mundial sobre seguridad alimentaria y cambio climático, y nuevas tecnologías que permitan producir más con menos impacto en el medio ambiente. El desafío de alimentar a 9.000 millones de personas para el ańo 2050 requiere un aumento del $70 \%$ en la producción de víveres. Pero ello no es sólo cuestión de incrementar la producción, sino que hacen falta cambios estructurales, para modificar el patrón de producción agrícola habrá que integrar los recursos naturales al modelo de desarrollo económico, más como bienes públicos globales que como medios ilimitados y disponibles en cualquier momento (ONU, 2011).

La Organización de Naciones Unidas para la Educación la Ciencia y la Cultura (2005) define el consumo sostenible como aquel modo de consumo de bienes y servicios que no dañan el medio ambiente ni la sociedad. Afirma que llevar un estilo de vida sostenible es de capital importancia para erradicar la pobreza y conservar y proteger el conjunto de recursos naturales del que dependen todas las formas de vida, y que la educación para el desarrollo sostenible promueve una ciudadanía responsable y lucha contra los efectos de los hábitos y estilos de consumo insostenibles sobre las sociedades y los recursos.

Camacho (2006) establece que para llevar a cabo cambios en los patrones de consumo es indispensable la solidaridad que daría algún sentido a la frugalidad, entendida esta como una disminución en el consumo individualista para hacer posible compartir con los demás lo que se puede adquirir. Esta combinación de solidaridad con frugalidad entendida de otra manera se aplicaría por lo menos de dos formas al cambio de patrones de consumo en los países en vías de desarrollo: 
1) Incrementando el consumo de bienes y servicios para la satisfacción de necesidades básicas en amplias capas de la población que carecen de lo mínimo.

2) Consumiendo en sociedad y no en el aislamiento individual. El ejemplo de la depresión originada en el consumo narcisista en los países desarrollados es un poderoso aliciente para evitar el egoísmo.

Sin embargo, ratifica que más allá de los cambios en patrones de consumo, los países subdesarrollados no tienen ningún futuro si no protegen sus recursos tanto de la destrucción ecológica como de la codicia ajena.

En el Manual de consumo sostenible en nuestra vida cotidiana (Confederación de Usuarios y Consumidores, 2006) aparecen los siguientes principios básicos:

1) Adquirir únicamente los productos que sean necesarios.

2) Obtener información de las condiciones sociales y medioambientales en que han sido elaborados los productos que vamos a obtener o los servicios que vamos a utilizar. Valorar las repercusiones medioambientales y sociales que puede tener el uso de un producto o servicio no solo en el ámbito local, sino también en su dimensión global.

3) Plantearnos las alternativas al comercio convencional, como el comercio justo, las inversiones socialmente responsables y la banca ética.

4) Exigir calidad en los bienes que adquirimos, practicando así un consumo crítico y responsable.

Todo ello perfila un modelo que apuesta por ser:
1) Un consumo crítico: los consumidores hemos de preguntarnos por las condiciones sociales y ecológicas en las que se elaboran los productos o servicios.

2) Un consumo ético: basado no en la satisfacción de los deseos personales sino en las opciones solidarias y respetuosas con el medio ambiente. Un modelo que propone la reducción del consumo como primer paso.

3) Un consumo que exija la responsabilidad social empresarial: las empresas no solo han de rendir cuentas en el plano económico, sino también en el social y en el medioambiental.

Sin embargo, el consumo consiente de productos menos tóxicos, menos explotadores y menos contaminantes que avance hacia un sistema económico que respete en lugar de explotar el medio ambiente tampoco es fácil. El creer que comprando productos amigables con el medio ambiente, verdes o más ecológicos no soluciona la totalidad de la grave situación en la que nos encontramos; se necesita mucho más que eso, se necesita consumir menos. El futuro que nos espera y les espera a nuestros nietos si no enfrentamos el presente al quedar sin recursos a la gente solo le quedaran tres opciones: sublevarse, emigrar o morir, y ninguna de las tres es aceptable (Leonard, 2010).

\section{Referencias}

I. Alien, F. (1964). Le only yesterday: an informal history of the nineteen-twenties. Nueva York.

2. Barber, B. (2008). Consumed: how markets corrupt children, infantilize adults, 
and swallow citizen whole. NuevaYork: Norton \& Co.

3. Bauman, Z. (2000). Trabajo, consumismo y nuevos pobres. Barcelona: Tedias.

4. Camacho, L. A. (2006, enero-agosto). Cambio en patrones de consumo. Revista de Filosofia de la Universidad de Costa Rica, XLIV, 49-56.

5. Consejo Empresarial Colombiano para el Desarrollo Sostenible (s. f.). Cecodes. Recuperado de http://www.cecodes. org.co/

6. Confederación de Usuarios y Consumidores (2006). Manual de consumo sostenible en nuestra vida cotidiana. Madrid: Confederación de Usuarios y Consumidores.

7. Comisario de Medio Ambiente, Comisión Europea (2008). La economía de los ecosistemas y la biodiversidad. Bélgica.

8. Cowdrick, E. (1927). The new economic gospel of comsumtion. Industrial Management.

9. Evaluación de los ecosistemas del milenio (2005). Estamos gastando más de lo que poseemos. ONU.

Io. Gutiérrez, J.; Capera, L. y Estrada, D. (2011). Reporte de establidad financiera. Bogotá: Banco de la República.

I I. Leonard, A. (2010). La historia de las cosas. Buenos Aires: Fondo de Cultura Económica.

I 2. Max-Neef, M. (1993). Desarrollo a escala humana. Barcelona: Icaria.

I 3. Moser, M.; Prentice, C. y Frazier, S. (1996). A global overview of wetland loss and degradation. En Conference Proceedings. Institute of International Education.
I4. New Economics Foundation (2012). Índice del planeta feliz.

I5. ONU (2011). Centro de Noticias ONU. Recuperado ede http:// www.un.org/spanish/News/story. asp?NewsID $=21717$

I6. Orr, D. (1999). The ecology of giving and consuming. En Rosenblatt (ed.). Consuming desires: consumption, culture, and the pursuit of happiness. Washington D.C.: Island Press.

17. Real Academia Española (1970). Diccionario de la lengua española (10. ${ }^{a}$ ed.). Madrid: Espasa Calpe.

I8. Real Academia Española (2005). Diccionario de la lengua española. Madrid: Santillana.

19. Rifkin, J. (2008). El fin del trabajo. Barcelona: Paidós.

20. Sachas, J. (2008). Economía para un planeta abarrotado. Barcelona: Debate.

2I. Seager, J. (1993). Coming to feminist terms with the global enviroment crisis. Earth Follies.

22. Speth, J. (2008). The bridge at the edge of the world. New Haven: Yale University Press.

23. Steeffen. (2005). The Nature of Global Change. Global Change IGB series.

24. Unesco (2005). Recuperado de http:// www.unesco.org/es/education-for-sustainable development/themes/sustainable-consumption/

25. Wilkinson, C. (2004). Status of coral reefs of the world. Australian: Institute of Marine Science, Townsville. 